(C) Springer Science+Business Media Singapore 2015

Tracey Wade Encyclopedia of Feeding and Eating Disorders 10.1007/978-981-287-087-2_33-1

\title{
Dissociation and Dissociative Disorders
}

Johan Vanderlinden ${ }^{1,2}$, Laurence Claes ${ }^{2,3}$, Kathleen De Cuyper ${ }^{1,2}$ and Elske Vrieze ${ }^{1}$

(1)

University Psychiatric Center, KU Leuven, Belgium

(2)

Faculty of Psychology and Educational Sciences, KU Leuven, Leuven, Belgium

(3)

Faculty of Medicine and Health Sciences (CAPRI), University Antwerp, Antwerp, Belgium

Johan Vanderlinden (Corresponding author)

Email: johan.vanderlinden@ppw.kuleuven.be

Laurence Claes

Email: laurence.claes@ppw.kuleuven.be

Kathleen De Cuyper

Email: kathleen.decuyper@ppw.kuleuven.be

Elske Vrieze

Email: elske.vrieze@uzleuven.be

Without Abstract

Synonyms

$\underline{\text { Trauma-induced dissociation and dissociative disorders; }}$ Disorders of extreme stress

\section{Definition and Diagnosis of Dissociation and Dissociative Disorders}

In the last decades, both the diagnosis and the treatment of dissociative disorders have received much attention in the field of mental disorders. According to DSM-5, the essential characteristic of dissociative disorders is a disruption in the usually integrated functions of consciousness, memory, identity, or perception of the environment. Dissociative symptoms are commonly encountered in people with a variety of psychiatric diagnoses and consist of experiences of derealization/depersonalization, identity confusion/fragmentation, and psychogenic amnesia as most specific characteristics. All dissociative disorders included in the DSM-5 refer to psychological dissociative manifestations and include the following diagnoses: dissociative identity disorder (DID), dissociative amnesia with or without dissociative fugue, depersonalization/derealization disorder, other specified dissociative disorder, and unspecified dissociative disorder. Patients with DID manifest the most severe level of dissociative symptoms. Curiously, DSM-5 only describes psychological aspects of dissociation. Somatoform dissociative symptoms are not addressed, i.e., dissociation which is manifested in a loss of the normal integration of somatoform components of experience (e.g., anesthesia and motor inhibition, taste and smell alterations). Nevertheless, several authors stress the multidimensional nature of dissociation, encompassing both psychological and somatoform symptoms. 
There is evidence that dissociative disorders are strongly related to a history of sexual, physical, and/or emotional abuse. For instance, the vast majority of DID patients report severe forms of abuse as well as highly invalidating family environments. It is hypothesized that dissociative symptoms in humans show some similarities with animal defensive responses to variable predatory imminence and injury. Hence, it has been argued that dissociation may develop as a psychological defense and survival mechanism activated when no escape to the traumatic event is possible. An individual who has experienced childhood trauma may therefore respond to emotions with behaviors that trigger avoidance, distraction, or dampening of emotions. According to the "escape theory" proposed by Heatherton and Baumeister, in eating disorders (ED), fasting and binge eating may have the function to avoid the confrontation with painful emotions and may facilitate the escape from awareness of threatening stimuli and emotions.

\section{Historical Background}

Dissociation is a descriptive concept introduced and developed by Pierre Janet at the end of the nineteenth century. In the nineteenth century, dissociation constituted the essential feature of a class of mental disorders called hysteria. In these hysterical patients, Janet described the splitting of "systems of ideas or "idées fixes (fixed ideas)" as an essential characteristic of dissociation and this process was evoked when confronted with a traumatic experience. At the end of the twentieth century, Janet's conceptions and ideas received new attention and the concept of dissociation and dissociative disorders were included for the first time as a distinct and separate diagnostic category in the DSM-III in 1980. The DSM-III created a separate category for the dissociative disorders and suggested criteria for a diagnosis of Multiple Personality Disorder giving legitimacy to the condition.

In recent years, Janet's theoretical assumptions have led to the development of the theory of trauma-related structural dissociation of the personality, a theory which is dominating the trauma field. According to the theory of structural dissociation, individuals who suffer from dissociative symptoms (or more complex trauma-related disorders) alternate between being fixated on their traumatic experiences and being detached from them. According to the theory of structural dissociation, this alternation directly reflects a failed integration between at least two different parts of the personality: an "emotional" part of the personality and an "apparently normal" part of the personality.

\section{Current Knowledge}

At the end of the last century, some case reports suggested the presence of dissociative symptoms in eating disorders. Next, several prevalence studies were carried out showing that dissociative symptoms are overrepresented in individuals with an ED with higher levels of dissociation being linked to more severe psychopathology. For example, in one of our first studies, approximately $13 \%$ of the ED patients reported pathological dissociative symptoms. Dissociation scores were comparable with the scores of patients with a dissociative disorder. Most of these ED patients received a Dissociative Disorder NOS diagnosis while DID was very rare. Dissociative symptoms were more prevalent in ED patients with a history of trauma and bingeing-purging behaviors. However, other researchers found no relationship between any form of child abuse and bulimic symptoms, whereas in the group of patients with bulimia nervosa, neglect and sexual abuse were correlated with dissociation. In other studies, robust associations were found between the presence of (somatoform) dissociative symptoms and bulimic symptoms such as bingeing episodes, laxative abuse, diet pill abuse, diuretic abuse 
and excessive exercise, as well as bulimic attitudes. These studies stress that the assessment of both psychological and somatoform dissociative symptoms is indicated in ED patients with bulimic features.

Some studies have focused on potential mediating factors between the trauma experience and the development of ED and dissociative symptoms. One study is suggesting that a negative body experience may mediate this link. Moreover, the negative body experiences were strongly related to the severity of abuse history (especially sexual abuse). The authors hypothesized that in traumatized ED patients, the disturbed body experience is probably not just related to a wish to be thin, but more to the wish to make the body unattractive or punish the body due to guilt feelings. This finding was confirmed in a large female sample of university students showing that body dissatisfaction was the only significant mediator of the relationship between trauma and binge eating.

Other studies found some support for the mediating role of dissociation in the relationship between childhood trauma and disordered eating. In AN and BN patients, it was found that dissociation significantly mediated the relationship between childhood sexual abuse and binge eating. Data from several other studies corroborate this finding showing again that dissociation was a significant mediator between childhood emotional abuse and eating psychopathology in a nonclinical female sample. Finally, one recent study found that both emotion dysregulation and dissociation were significant mediators between childhood trauma and eating psychopathology. Hence, the majority of these studies suggest a mediating role of dissociation between childhood trauma and eating psychopathology, especially binge eating and purging behaviors. However, we have to notice that one study found no evidence for the role of dissociation in the link between the presence of childhood emotional abuse and unhealthy eating behaviors in a female student sample.

Recently, some studies were carried out in patients with binge eating disorder (BED). The results showed a positive association between trauma, dissociative symptoms, and BED. One of the first studies highlighted that obese subjects with a diagnosis of BED had higher scores on both early traumatic experiences and dissociation, than obese subjects without BED, and that dissociation was significantly associated with traumatic experiences. Additionally, it was found that the presence of childhood sexual abuse was the strongest predictive factor of binge eating episodes and that dissociation fully mediated the relationship between childhood abuse and the severity of binge eating symptoms. The authors also indicated that negative affect and dissociation were elevated prior to binge eating episodes. In addition, antecedent effects of dissociation on binge eating were independent of those attributable to negative affect. In agreement to the theory of escape from self-awareness, it is hypothesized that when negative emotional states are activated, a shift toward lower levels of cognition and self-awareness is initiated, which involves cognitive processes similar to dissociation. This mechanism tends to remove the inhibitions, thereby facilitating the start of binge eating or overeating, both in clinical and in nonclinical subjects. Finally, one study is demonstrating that it is also important to assess somatoform dissociative symptoms in patients with BED. The results suggest that the relationship between dissociation and binge eating is primarily related to the somatic symptoms of dissociation that reflect disruptions to body-based awareness and bodily functions. These findings seem to confirm results from studies carried out in patients with BN and they underline the importance of evaluating also somatoform dissociation in patients with binge eating.

Finally, also in eating disorders, higher levels of dissociation have been demonstrated to predict lower effectiveness of treatments, such as cognitive-behavioral therapy. This moderation effect of dissociation on treatment outcome has also been observed in other psychiatric disorders, such as anxiety disorders and obsessive-compulsive disorder. Several authors suggest that the fragmentation of consciousness, identity and memory which are key 
aspects of dissociation, interfere with emotional learning processes and thereby limit the effectiveness of treatment. Moreover, during psychotherapy, detachment and lowering the level of awareness are the reactions of dissociation prone patients when they are confronted with the powerful emotions which go along with the treatment. These reactions are also thought to interfere with treatment response. Therefore, during the treatment of eating disorder patients, dissociation has to be dealt with directly.

\section{Current Treatment Approaches}

From a clinical viewpoint we try to understand the interplay between trauma, dissociative symptoms, and ED in a more functional - rather than causal - way. Clinical experience is indeed showing that the eating disorder symptoms in these patients may have different functions. Perhaps the abusive experience has reshaped an already developing or existing ED into a more self-destructive way of life. Or perhaps the victim "learned" to dissociate and to use bingeing and purging as an escape or avoidance strategy for feelings, memories, sensations, and cognitions linked to the trauma. It is hypothesized that the thinness/fatness can function as a way to make the body sexually unattractive; vomiting and purging can be used as a way to express feelings of disgust, dirtiness, the need to purify the body, or to punish oneself. Hence, the ED symptoms can be considered as a way to cope with the emotional consequences of the trauma and become a survival strategy in many patients! These are the types of hypotheses that guide our psychotherapeutic work, the basic principles of which will now be discussed.

It is clear that no evidence-based approach exists for the treatment of dissociative disorders and/or dissociative symptoms in ED patients. However, a "consensus" exists between the leading clinicians and experts in the field regarding the necessary steps and treatment phases. At present, standards for assessment and treatment of dissociative conditions can be found in the International Treatment Guidelines of the International Society for the Study of Trauma Dissociation (ISSTD), now in the third edition ( 2011$)$. They summarize expert consensus concerning effective assessment and treatment for patients suffering from dissociation. Meanwhile, they present key findings and generally accepted principles that reflect current scientific knowledge and clinical experience specific to the diagnosis and treatment of dissociative disorders. Many of these principles can be included and/or combined with the existing evidence-based treatments for eating disorders.

As a first step, a careful clinical interview is needed. This step must include some assessment of both psychological and somatoform dissociation and an evaluation of the presence of traumatic experiences. This is also recommended in eating disorder patients especially those patients with bingeing-purging behaviors.

Nowadays, a consensus between experts exists about the fact that complex trauma-related disorders must be treated in sequenced stages. Hence, the treatment will commonly consist of three different phases or stages:

1 .

Establishing safety, stabilization, and symptom reduction

2.

Confronting, working through, and integrating traumatic memories

3.

Identity integration and rehabilitation

1 .

In the initial phase of treatment, emphasis should be placed on establishing a therapeutic alliance, educating patients about diagnosis and symptoms, and explaining the process of 
treatment. Following the International Treatment Guidelines of the ISSTD ( $\underline{2011}$ ), the goals of Phase 1 treatment include maintaining personal safety, controlling symptoms, modulating affect, building stress tolerance, enhancing basic life functioning, and building or improving relational capacities.

In eating disorder patients with complex trauma and dissociation, special attention must be paid to the development of a trustful therapeutic alliance, which can be very challenging not only in the beginning but also in later phases of treatment. Another basic element in the treatment is to educate patients (and families) about dissociation and related impulsive behaviors. Many patients believe they are "weird," especially when they reveal hearing voices in their head, cutting themselves, bingeing and vomiting in secret, etc. Compared to the ED patients without complex trauma and dissociation, the therapist must be aware that both the weight loss and/or bingeing/purging behaviors may have the function of avoiding confrontation with painful emotions and memories rather than to avoid the confrontation with a normal body weight. Nevertheless, the therapist must try to motivate and help the patient to normalize eating habits and weight and to stop "step by step" the eating disorder symptoms. In the case of complex trauma, this part of the treatment can be very challenging and more complex compared to the ED patients without a trauma history. The therapist will have to focus not only on the typical eating disorder symptoms such as the bingeing and purging behaviors but also on a wide variety of impulsive behaviors. Many patients have to find a way to regain control over the dissociative episodes frequently linked to impulsive and selfdestructive behaviors. They often believe they are out of control. Therefore, a basic treatment goal is to reach sufficient "internal control" over what they do.

In this initial phase, the patient is invited by means of self-observation to become aware of the different stimuli that may trigger dissociative mental states in which they risk to lose control. At the same time, in order to acquire more self-mastery and reorienting in the "here and now" situation, both behavioral (jogging, yoga, writing, singing, asking for help, etc.) and emotional strategies (self-hypnosis, heart coherence training, mindfulness, listening to music, etc.) are instructed. Especially in the case of self-destructive behavior, a plan for future protection must be prepared together with the patient. The patient needs to develop insight in the triggers and consequences of the self-harming behaviors, and look for alternative behaviors which are less harmful. In the selection of these alternative behaviors, it is important that they are easily accessible, do not hurt themselves or others, and lead to emotional release (e.g., chatting with friend, running, taking a shower) (Claes and Muelhlenkamp 2014). Another important step in the establishment of safety may be the involvement of meaningful others in the treatment, such as family members, partner, or friends. If patients live in social isolation, it is important to either provide a social network (e.g., support group) or help the patients to create one, so that they can learn to trust other people outside the therapeutic milieu and ask for support. At the same time they have to find out what type of milieu or people are a potential source of danger.

2 .

Only when sufficient internal control has been established and important progress has been made regarding the eating disorder symptoms and other impulsive behaviors, the patient can move to the second phase of treatment namely "confronting, working through, and the integration of traumatic memories." Many evidence-based treatments such as Eye Movement Desensitization and Reprocessing (EMDR) have been developed and they all share the same treatment principles. All trauma treatments are phase oriented and will in a first step teach the patient some specific skills to master the level of anxiety. In a second step, some exposure to important anxiety-provoking stimuli takes place following a specific treatment protocol with regular assessment of the level of negative emotions. Exposure goes on until some 
desensitization of negative affect takes place as expressed by the subjective evaluation of the patient. Meanwhile new adequate cognitive schemas will be installed.

3.

However, we have to remark that many patients will skip this second phase and move, once sufficient internal control has been established, to phase 3: "Identity integration and rehabilitation." In this phase, the patient is starting a new life while trying to further stabilize the therapeutic progress. Often patients will grieve because of the "lost" childhood while at the same time going back to or starting a "normal" life. The therapist will help the patient to gain more confidence and self-esteem and to take an active role in creating an environment in which trust, self-care, autonomy, and intimacy can flourish.

\section{Future Directions}

Research is clearly showing that abusive experiences increase the vulnerability in young women to develop an ED, in particular one with bingeing-purging characteristics. This subgroup of ED patients will often be confronted with both psychological and somatoform dissociative experiences. It is hypothesized based on clinical experiences that the ED symptoms in this subsample may function as a survival strategy while research findings show a mediating role for dissociation in the association between trauma and ED. Based on these findings and hypotheses, clinicians must become more aware of the different pathways that may lead to the development of an ED and realize that different pathological pathways leading to eating disorders may deserve different therapeutic approaches. We are missing evidence for matching patients to treatments, but we recommend that the evidence from the psycho-trauma field needs to be integrated within the ED treatment, when indicated.

\section{Cross-References}

- Body Image

- Coping: Escape Avoidance

- Course and Outcome of Eating Disorders

- Emotion Regulation Difficulties

- Intensive Treatments

- Treatment-matching: Eating Disorder Diagnosis and Selection of the Best $\underline{\text { Treatment }}$

- Weight and Shape Concern and Body Image

References and Further Reading

Claes, L., \& Muehlenkamp, J. J. (Eds.). (2014). Non-suicidal self-injury in eating disorders. Heidelberg: Springer.

Dalle Grave, R., Oliosi, M., Todisco, P., \& Vanderlinden, J. (1997). Self-reported traumatic experiences and dissociative symptoms with and without binge-eating disorder. Eating Disorders, 5, 105-109. CrossRef

Heatherton, T. F., \& Baumeister, R. F. (1991). Binge eating as an escape from self-awareness. Psychological Bulletin, 110, 86-108. CrossRefPubMed 
International Society for the Study of Trauma and Dissociation. (2011). Guidelines for treating dissociative identity disorder in adults, third revision. Journal of Trauma \& Dissociation, 12, 115-187. CrossRef

La Mela, C., Maglietta, M., Lucarelli, S., Mori, S., \& Sassaroli, S. (2013). Pretreatment outcome indicators in an eating disorder outpatient group: The effects of self-esteem, personality disorders and dissociation. Comprehensive Psychiatry, 54, 933-942.

\section{CrossRefPubMed}

Moulton, S. J., Newman, E., Power, K., Swanson, V., \& Day, K. (2015). Childhood trauma and eating psychopathology: A mediating role for dissociation and emotion dysregulation? Child Abuse and Neglect, 39, 167-174. CrossRefPubMed

Van der Hart, O., Nijenhuis, E. R. S., \& Steele, K. (2006). The haunted self: Chronic traumatization and structural dissociation of the personality. New York/London: Norton. Vanderlinden, J., Vandereycken, W., Van Dyck, R., \& Vertommen, H. (1993). Dissociative experiences and trauma in eating disorders. International Journal of Eating Disorders, 13, 187-194. CrossRefPubMed

Vanderlinden, J., Vandereycken, W., \& Claes, L. (2007). Trauma, dissociation and impulse dyscontrol: Lessons from the eating disorders field. In E. Vermettten, M. Dorahy, \& D. Spiegel (Eds.), Dissociation: Neurobiology and treatment (pp. 317-333). Arlington: American Psychiatric Publishing. Waller, G., Babbs, M., Wright, F., Potterton, C., Leyer, C., \& Leubg, N. (2003). Somatoform dissociation in eating-disordered patients. Behaviour Research and Therapy, 41, 619-627. CrossRefPubMed 